\title{
INSUFICIÊNCIA RESPIRATÓRIA
}

\author{
RESPIRATORY FAILURE
}

Adriana Inacio de Pádua ${ }^{1}$; Flávia Alvares ${ }^{1}$ \& José Antônio Baddini Martinez²

${ }^{1}$ Médica Assistente. ${ }^{2}$ Docente. Divisão de Pneumologia. Departamento de Clínica Médica. Faculdade de Medicina de Ribeirão Preto - USP. CorResPondÊnCIA: José Antônio Baddini Martinez. Departamento de Clínica Médica. Hospital das Clínicas de Ribeirão Preto. Avenida Bandeirantes 3900, CEP 14048-900, Ribeirão Preto - SP, Brasil. Telefone: 16-6022531; Fax: 16-6336695; email: jabmarti@fmrp.usp.br

PÁDUA Al; ALVARES F \& MARTINEZ JAB. Insuficiência respiratória. Medicina, Ribeirão Preto, 36: 205-213, abr./dez. 2003.

RESUMO - O conceito de insuficiência respiratória (IR) compreende a dificuldade encontrada pelo Sistema Respiratório em desempenhar adequadamente sua principal função, ou seja, a promoção das trocas gasosas. Por ser decorrente de várias condições, pode apresentar-se, clinicamente, de forma muito variada. Seu diagnóstico depende da análise dos níveis de oxigênio e gás carbônico através da gasometria arterial. O presente artigo fornece uma visão geral da fisiologia das trocas gasosas e suas alterações, conceitos fundamentais para a compreensão da classificação e mecanismos envolvidos nos diferentes tipos de insuficiência respiratória. São ainda enfatizados aspectos gerais, relacionados com a terapêutica, na forma de noções sobre oxigenioterapia e indicações de suporte ventilatório.

UNITERMOS - Insuficiência Respiratória. Troca Gasosa Pulmonar. Respiração Artificial.

\section{1- DEFINIÇÃO}

A insuficiência respiratória (IR) pode ser definida como a condição clínica na qual o sistema respiratório não consegue manter os valores da pressão arterial de oxigênio $\left(\mathrm{PaO}_{2}\right)$ e/ou da pressão arterial de gás carbônico $\left(\mathrm{PaCO}_{2}\right)$ dentro dos limites da normalidade, para determinada demanda metabólica. Como a definição de IR está relacionada à incapacidade do sistema respiratório em manter níveis adequados de oxigenação e gás carbônico, foram estabelecidos, para sua caracterização, pontos de corte na gasometria arterial, como se segue:

- $\mathrm{PaO}_{2}<60 \mathrm{mmHg}$

- $\mathrm{PaCO}_{2}>50 \mathrm{mmHg}$

A IR pode ser classificada quanto à velocidade de instalação, em aguda e crônica. Na IR aguda, a rápida deterioração da função respiratória leva ao surgimento de manifestações clínicas mais intensas, e as alterações gasométricas do equilíbrio ácido-base, alcalose ou acidose respiratória, são comuns. Quando as alterações das trocas gasosas se instalam de maneira progressiva ao longo de meses ou anos, estaremos diante de casos de IR crônica. Nessas situações, as manifestações clínicas podem ser mais sutis e as alterações gasométricas do equilíbrio ácido-base, ausentes. Exemplos de tal condição são a doença pulmonar, obstrutiva, crônica (DPOC), avançada. Vale salientar que quadros de IR aguda podem instalar-se tanto em indivíduos previamente sadios como, também, sobrepor-se à IR crônica, em pacientes com processos de longa data. Nessa última situação, o uso do termo IR crônica, agudizada é aceitável.

\section{2- TROCAS GASOSAS}

Uma vez que o conceito de IR baseia-se no comportamento das trocas gasosas, é essencial o co- 
nhecimento de sua fisiologia. Embora as trocas gasosas sejam um processo contínuo, podem ser divididas, para fins didáticos, em quatro fases.

\section{Ventilação}

Processo cíclico, responsável pela renovação do gás alveolar. Resulta da ação integrada entre o centro respiratório, localizado no sistema nervoso, central (SNC), vias nervosas, caixa torácica (estrutura osteomuscular) e os pulmões. A ventilação alveolar é dada pela seguinte fórmula:

$$
\mathbf{V A}=(\mathbf{V T}-\mathrm{VD}) \mathbf{f}
$$

Onde:

$$
\mathrm{VA}=\text { ventilação alveolar. }
$$

$\mathrm{VT}=$ volume corrente.

$\mathrm{VD}=$ volume do espaço morto, anatômico.

$\mathrm{f}=$ freqüência respiratória.

Alterações da ventilação podem ocorrer de forma localizada ou global, levando a prejuízo da lavagem do gás carbônico e ao aporte de oxigênio. Tais distúrbios podem se instalar devido a:

- Alterações regionais ou difusas da elasticidade pulmonar, como ocorre no enfisema.

- Obstruções regionais ou difusas, inclusive aquelas que envolvem as pequenas vias aéreas, como ocorre na DPOC e na asma.

- Modificações do espaço morto anatômico e/ou do espaço morto do compartimento alveolar, que pode ser observado, por exemplo, na DPOC.

- Modificações da expansibilidade pulmonar, secundárias à presença de exsudatos, tumores ou fibrose nas paredes alveolares ou interstício pulmonar.

- Hipoventilação por comprometimento do sistema nervoso, músculos efetores ou deformidades da caixa torácica.

\section{Perfusão}

A circulação pulmonar é munida de um vasto leito vascular, no qual os pequenos vasos e os capilares são os responsáveis pela principal atividade funcional. Tem características de um sistema hidráulico de baixa pressão, complacente e de baixa resistência, que podem ser modificadas por fatores intrínsecos (pressão, volume, fluxo) e extrínsecos (inervação autonômica, controle humoral) e pelos gases respiratórios.

A perfusão pulmonar pode ser alterada por diferentes desarranjos, como os indicadores:

- Obstrução intraluminal: doenças tromboembólicas, vasculites, acometimento vascular por colagenoses, etc.
- Redução do leito vascular: enfisema, ressecção do parênquima pulmonar, etc.

- Colabamento vascular por hipotenção e choque; compressão vascular por lesões tumorais ou aumento da pressão alveolar, como no caso do uso de ventiladores com pressão positiva.

\section{Relação ventilação/perfusão (V/Q)}

Os valores finais da $\mathrm{PaO}_{2}$ e $\mathrm{PaCO}_{2}$ resultam de interações entre a taxa de ventilação alveolar e o respectivo fluxo sangüíneo. Mesmo em indivíduos normais, a relação V/Q não é uniforme em todo o pulmão, sendo maior nos ápices. As situações polares dos distúrbios V/Q são representadas por alvéolos ventilados, mas não perfundidos (espaço morto) e pela situação oposta, alvéolos não ventilados mas perfundidos adequadamente (shunt). Entre esses pontos de alterações extremas, encontramos situações em que ocorrem alvéolos bem ventilados, mas pouco perfundidos (efeito espaço morto) ou então alvéolos com ventilação reduzida e perfusão sangüínea mantida (efeito shunt).

De um ponto de vista, prático, alterações da relação V/Q são as causas mais comuns de distúrbios das trocas gasosas. Quadros graves de hipoxemia arterial, como aqueles observados em pneumonias extensas ou na SARA, são conseqüência da presença de áreas de shunts e efeito shunt no nível do parênquima pulmonar.

\section{Difusão}

A capacidade de difusão pulmonar pode ser definida como a quantidade de gás transferida por minuto, através da membrana alveolocapilar, para cada milímetro de mercúrio de diferença entre as pressões parciais deste gás no espaço alveolar e no sangue. $\mathrm{O}$ fluxo de oxigênio é dirigido pela maior pressão no nível alveolar em relação ao sangue capilar. Diferentes fatores podem influenciar a capacidade de difusão pulmonar, alterando, assim, os valores dos gases sangüíneos. Entre eles, vale a pena citar: espessura da membrana alveolocapilar e distância de difusão; extensão da superfície da membrana de difusão; solubilidade dos gases; propriedades de difusibilidade do meio e alterações dos gradientes de pressão dos gases. Um exemplo de alteração da espessura e da composição da membrana alveoloarterial é a doença intersticial, pulmonar, que frequientemente culmina com a instituição de fibrose intersticial. Exemplo clássico de redução da superfície da membrana alveoloarterial é o enfisema. De modo geral, alterações da difusão pul- 
monar só levam a distúrbios das trocas gasosas em situações de sobrecarga respiratória, como durante a realização de exercícios.

\section{Gradiente alveoloarterial}

O grau da eficácia global das trocas gasosas pode ser avaliado à beira do leito por uma série de cálculos matemáticos, simples, entre eles a determinação da diferença entre a pressão de oxigênio do alvéolo $\left(\mathrm{PAO}_{2}\right)$ e a pressão de oxigênio arterial $\left(\mathrm{PaO}_{2}\right)$. Abreviado como $\mathrm{P}(\mathrm{A}-\mathrm{a}) \mathrm{O}_{2}$, o gradiente alveoloarterial de oxigênio é, normalmente, pequeno, entre 5 e 10 $\mathrm{mmHg}$, alargando-se na presença de IR. Além disso, ele inclui, em seu cálculo, os valores da $\mathrm{PaCO}_{2}$, não sendo, assim, influenciado pela presença de hipoventilação. Dessa forma, o cálculo da $\mathrm{P}(\mathrm{A}-\mathrm{a}) \mathrm{O}_{2}$ é um método mais preciso para a caracterização da presença de IR. Além disso, a diferenciação entre a presença de alterações da relação V/Q ou shunt pode ser feita pela administração de oxigênio a $100 \%$ ao paciente. Uma boa resposta ao oxigênio indica desproporção V/Q como causa da hipoxemia, enquanto que, na presença de shunt verdadeiro, a hipoxemia se mantém. Vale notar que são disponíveis equações para o cálculo do shunt real. O gradiente alveoloarterial, por sua vez, é calculado pela seguinte equação:

$$
\begin{aligned}
& \mathbf{P}(\mathrm{A}-\mathrm{a}) \mathrm{O}_{2}=\left[\mathrm{FiO}_{2}\left(\mathrm{P}_{\mathrm{B}}-47\right)-\left(\mathrm{PaCO}_{2} / \mathrm{R}\right)-\mathrm{PaO}_{2}\right] \\
& \text { Onde: } \\
& \mathrm{FiO}_{2}=\text { fração inspirada de oxigênio. } \\
& \mathrm{P}_{\mathrm{B}}=\text { pressão barométrica local. } \\
& 47=\text { pressão de vapor de água nas } \\
& \mathrm{R}=\text { quociente respiratório, habitual- }
\end{aligned}
$$

\section{3- CLASSIFICAÇÃO}

A IR, classicamente, é classificada em tipo I (hipoxêmica) e tipo II (hipercápnica). Uma lista de causas selecionadas de IR está contida na Tabela I.

Na IR tipo I, também chamada de alveolocapilar, os distúrbios fisiopatológicos levam à instalação de hipoxemia, mas a ventilação está mantida. Caracterizase, portanto, pela presença de quedas da $\mathrm{PaO}_{2} \mathrm{com}$ valores normais ou reduzidos da $\mathrm{PaCO}_{2}$. Nesses ca-
Tabela I - Causas de Insuficiência Respiratória.

- SARA

$$
\text { Insuficiência Respiratória do Tipo I }
$$

- Pneumonias

- Atelectasias

- Edema Pulmonar

- Embolia Pulmonar

- Quase afogamento

- DPOC em exacerbação

- Asma grave

- Pneumotórax

\section{Insuficiência Respiratória do Tipo II}

\section{1- Alterações do SNC}

- Lesões estruturais (neoplasia, infarto, hemorragia, infecção).

- Drogas depressoras.

- Hipotireoidismo.

- Alcalose metabólica.

- Apnéia do sono central.

- Doenças da medula: trauma raquimedular;neoplasia; infecção; infarto; hemorragia; mielite transversa; GuilainBarré; esclerose lateral, amiotrófica; etc.

2- Alterações neuromusculares, periféricas

- Doenças causadas por neurotoxinas: tétano, botulismo, difteria.

- Miastenia gravis.

- Síndromes paraneoplásicas: Eaton Lambert.

- Distúrbios eletrolíticos : hipofosfatemia, hipomagnesemia, hipocalemia, hipocalcemia.

- Distrofias musculares

- Poliomiosites.

- Hipotireoidismo.

- Miosite infecciosa.

3- Disfunção da parede torácica e pleura

- Cifoescoliose.

- Espondilite Anquilosante.

- Obesidade.

- Tórax instável.

- Fibrotórax.

- Toracoplastia.

4- Obstrução das vias aéreas, superiores

- Epiglotite.

- Edema de laringe.

- Aspiração de corpo estranho.

- Paralisia de cordas vocais, bilateralmente.

- Estenose de traquéia, traqueomalácia.

- Tumores nas vias aéreas, superiores.

- Apnéia do sono, obstrutiva. 
sos observa-se elevação do gradiente alveoloarterial de oxigênio devido a distúrbios da relação V/Q. Compreende doenças que afetam, primariamente, vasos, alvéolos e interstício pulmonar. Exemplo dessas condições seriam casos de pneumonias extensas ou da síndrome da insuficiência respiratória aguda (SARA).

Nos casos de IR tipo II, ocorre elevação dos níveis de gás carbônico por falência ventilatória. Além disso, também é comum hipoxemia em pacientes, respirando ar ambiente. Esse tipo de IR também é chamado de insuficiência ventilatória. Pode estar presente em pacientes com pulmão normal como, por exemplo, na presença de depressão do SNC e nas doenças neuro-musculares. Entretanto, freqüentemente, sobrepõe-se a casos de IR tipo I, quando a sobrecarga do trabalho respiratório precipita a fadiga dos músculos respiratórios.

O cálculo do gradiente alveoloarterial de oxigênio permite diferenciar os tipos de IR. Hipoxemia com gradiente aumentado indica defeito nas trocas alveolocapilares e aponta para IR tipo I. Hipoxemia com gradiente normal é compatível com hipoventilação alveolar (IR tipo II).

\section{4- QUADRO CLÍNICO}

Uma vez que as causas e os mecanismos envolvidos com a sua gênese são diversos, a apresentação clínica de casos com IR pode ser muito variada. Entretanto, alguns sintomas e sinais são bastante comuns, independente da etiologia, e relacionam-se, principalmente, com as alterações observadas dos gases sangüíneos.

Pacientes com IR, habitualmente, queixam-se de dispnéia e demonstram elevações das freqüências respiratória e cardíaca. Cianose está igualmente presente, quando as concentrações sangüíneas da hemoglobina reduzida excederem $5 \mathrm{~g} / \mathrm{dl}$. A medida que a hipoxemia acentua-se, manifestações neurológicas, tais como diminuição da função cognitiva, deterioração da capacidade de julgamento, agressividade, incoordenação motora e mesmo coma e morte, podem surgir. Manifestações semelhantes podem ser causadas por elevações agudas do gás carbônico. Nos casos em que há hipoxemia crônica, os pacientes podem apresentar sonolência, falta de concentração, apatia, fadiga e tempo de reação retardado. A hipercapnia crônica pode desencadear sintomas semelhantes aos da hipoxemia crônica, além de cefaléia, particularmente matinal, distúrbios do sono, irritabilidade, insatisfação, sonolência, coma e morte.
As manifestações cardiovasculares da hipoxemia e elevação do gás carbônico incluem elevações iniciais da frequiência cardíaca, do débito cardíaco e vasodilatação arterial difusa, seguidos por depressão miocárdica, bradicardia, choque circulatório, arritmias e parada cardíaca.

\section{5- DIAGNÓSTICO}

O diagnóstico e a investigação da causa da IR baseia-se numa história clínica informativa, exame físico, detalhado e exames complementares, adequados.

A história clínica, obtida do paciente ou acompanhantes, deverá obrigatoriamente pesquisar, além da queixa ou queixas atuais do doente, a ocorrência de sintomas semelhantes previamente, a presença de doenças de base, antecedentes pessoais, e o uso, atual ou anterior, de medicações com atuação no aparelho respiratório e SNC.

O exame físico do tórax deve ser detalhado, envolvendo, além de percussão e ausculta, ocorrência de cornagem, análise do padrão respiratório, presença de enfisema subcutâneo, tiragem, uso de músculos acessórios da respiração e presença de movimento paradoxal, do abdômen. A presença na inspiração de assincronia toracoabdominal, com expansão do tórax e retração simultânea das porções superiores da parede abdominal, significa fadiga diafragmática e risco de apnéia eminente, sendo indicação para instalação de ventilação mecânica.

A confirmação da presença de IR só é feita pela análise dos gases sangüíneos. Uma indicação rápida das condições das trocas gasosas é dada pela oximetria de pulso. Uma $\mathrm{SaO}_{2}$ inferior a $90 \%$ é fortemente indicativa do diagnóstico. Entretanto, inúmeros fatores podem influenciar a leitura desses equipamentos, gerando leituras errôneas, entre elas, a presença de choque circulatório, má perfusão tecidual, cor da pele, etc. Além disso, a oximetria de pulso não fornece medidas relativas aos níveis de gás carbônico. Dessa forma, a colheita de uma gasometria arterial é obrigatória.

De modo geral, considera-se uma troca gasosa inadequada, quando a $\mathrm{PaO}_{2}$ é menor que $60 \mathrm{mmHg}$, ou, ainda, quando a $\mathrm{PaCO}_{2}$ ultrapassa $45 \mathrm{mmHg}$. Porém, cuidados devem ser tomados na interpretação da gasometria arterial:

- Os valores que definem IR são válidos para indivíduos respirando ar ambiente no nível do mar. Moradores onde há grandes altitudes apresentam níveis menores de oxigenação sangüínea, porém sem sintomas. 
- Valores confiáveis de gases arteriais só são obtidos com gasometrias colhidas, pelo menos, vinte minutos após a mudança da $\mathrm{FiO}_{2}$, administração de medicações inalatórias ou procedimentos fisioterápicos. A amostra sanguiínea deve ser prontamente analisada após sua colheita e transportada ao laboratório, em gelo. Os resultados de gasometria analisada uma hora após a colheita, ainda que mantida a baixas temperaturas, não espelham as reais condições do paciente.

- É fundamental saber com que fração inspirada de oxigênio foi colhida a gasometria. Uma $\mathrm{PaO}_{2}$ normal, mantida às custas de suplementação com altos fluxos de oxigênio é naturalmente insatisfatória. Dessa forma, IR pode ser igualmente caracterizada na presença de uma relação $\mathrm{PaO}_{2} / \mathrm{FiO}_{2}$ inferior a 300, onde $\mathrm{FiO}_{2}$ corresponde à fração inspirada de oxigênio, em números absolutos (por exemplo, ar ambiente $=0,21$ ).

- Os níveis de oxigenação devem ser interpretados em função da idade. Indivíduos idosos fisiologicamente são mais hipoxêmicos do que jovens. Uma estimativa da $\mathrm{PaO}_{2}$ prevista para a idade, pode ser obtida pela equação:

\section{$\mathrm{PaO}_{2}=[$ 96,2 $-(0,4 \mathrm{X}$ idade em anos $)]$}

Do mesmo modo, a $\mathrm{P}(\mathrm{A}-\mathrm{a}) \mathrm{O}_{2}$ média, prevista para a idade pode ser estimada pela fórmula:

$$
\mathrm{P}(\mathrm{A}-\mathrm{a}) \mathrm{O}_{2}=[(\text { idade em anos/4) }+4]
$$

- O parâmetro gasométrico que melhor se correlaciona com a ventilação alveolar é a $\mathrm{PaCO}_{2}$, a qual é medida diretamente pela gasometria arterial ou estimada pela capnografia.

- Pacientes portadores de pneumopatias crônicas, tais como DPOC, podem apresentar, cronicamente, níveis acentuados de hipoxemia e hipercapnia, em condições basais. Nesses indivíduos, a caracterização gasométrica de uma descompensação aguda pode ser feita, comparando-se os valores dos gases de momento com exames colhidos em momentos de estabilidade clínica. Além disso, deve-se observar atentamente o $\mathrm{pH}$ arterial. A presença de alcalose respiratória pode indicar hiperventilação por acentuação da hipoxemia, enquanto a acidose respiratória indica retenção aguda de gás carbônico.

- Pacientes com quadros de crises asmáticas, graves podem mostrar elevações transitórias dos níveis da $\mathrm{PaCO}_{2}$ devido a broncoespasmo muito intenso e alterações da relação V/Q. Tais pacientes devem ser tratados agressivamente e monitorados de perto, tanto clínica como laboratorialmente, devido à possibilidade de a retenção do gás carbônico agravar-se por instalação de fadiga muscular, respiratória.

- Pacientes com quadros neuromusculares podem apresentar hipoxemia e elevações acentuadas do gás carbônico com pH arterial, normal. Isso ocorre, quando o ritmo de instalação da IR for muito lento. Nessas condições, a indicação de suporte respiratório deve basear-se, também, em outros critérios, tais como presença de sintomas clínicos e alterações espirométricas (capacidade vital, forçada, geralmente abaixo de $40 \%$ ).

Em casos de IR, é obrigatória a realização de radiografias de tórax em projeções postero-anterior e perfil, visando detectar a presença de alterações pulmonares. Exames adicionais, tais como fibrobroncoscopias, eletrocardiograma, ecocardiograma, tomografia de tórax e culturas, poderão ser pedidos em função das suspeitas e do rumo da investigação clínica.

\section{6- TRATAMENTO}

O tratamento da IR deve ser individualizado, em função das causas desencadeantes e dos mecanismos fisiopatológicos, envolvidos. Broncodilatadores, corticosteróides, diuréticos, antibióticos e procedimentos cirúrgicos poderão ser de maior ou menor valia, em função das condições de base. Apesar disso, alguns princípios gerais se aplicam à maioria dos casos.

\section{Manutenção das vias aéreas}

A manutenção de vias aéreas pérvias e a profilaxia de complicações, em especial aspiração, são de fundamental importância em pacientes com IR, particularmente naqueles com distúrbios da consciência. Nesse caso, o paciente deve ser colocado em decúbito lateral com a cabeça abaixada e a mandíbula puxada para frente, visando evitar a obstrução pela língua. Com essa manobra, não raro, faz-se o diagnóstico de obstrução alta por vômito ou corpo estranho e pode providenciar-se a desobstrução.

O uso de cânula orofaríngea (chupeta) é adequado, quando se espera o rápido retorno da consciência, como, por exemplo, na recuperação anestésica. Caso se espere uma inconsciência mais prolongada ou ventilação mecânica seja necessária, a entubação endotraqueal está indicada. Em casos de obstrução 
alta acima das cordas vocais, a realização de cricotireoidotomia ou traqueostomia poderá ser necessária.

Pacientes com entubação traqueal ou traqueostomia, particularmente quando sedados ou em coma, devem ter suas vias aéreas periodicamente aspiradas, para evitar obstruções. Frente a dificuldades de ciclagem de um respirador mecânico, caracterizadas por freqüência respiratória, elevada, volume corrente baixo e/ou picos de pressão inspiratória, excessivos, devese pensar na possibilidade de obstrução da luz do tubo por rolha de catarro.

\section{Oxigenoterapia}

A administração de oxigênio estará indicada nos casos de IR aguda, quando a $\mathrm{PaO}_{2}$ for inferior a 60 mmHg ou a $\mathrm{SaO}_{2}$ inferior a $90 \%$. Nos casos de IR crônica, onde a tolerância à hipoxemia é maior, podese utilizar uma $\mathrm{PaO}_{2}$ limiar de $55 \mathrm{mmHg}$. Nessas condições, a oxigenoterapia sempre deverá ser introduzida, particularmente, nos casos de IR tipo I.

Os objetivos clínicos, específicos da oxigenioterapia são:

1- corrigir a hipoxemia aguda, suspeita ou comprovada;

2- reduzir os sintomas associados à hipoxemia crônica;

3- reduzir a carga de trabalho que a hipoxemia impõe ao sistema cardiopulmonar.

Existe uma grande variedade de dispositivos fornecedores de oxigênio, capazes de liberar uma ampla gama de valores de $\mathrm{FiO}_{2}$. Alguns sistemas são desenhados para fornecer uma $\mathrm{FiO} 2$ fixa, enquanto outros fornecem valores variáveis, não apenas em função da regulação do fluxo de gás, como, também, do padrão respiratório apresentado pelos pacientes. A administração de oxigênio pode dar-se por três grandes grupos de sistemas: os de baixo fluxo, os sistemas com reservatório e os de alto fluxo. Exemplos de dispositivos de baixo fluxo são as cânulas e os cateteres nasais. Exemplos de sistemas com reservatório são as máscaras simples e as máscaras com bolsas. Exemplos de sistemas de alto fluxo são as máscaras de Venturi, os nebulizadores e os assim chamados "tubos T". Cada dispositivo fornecedor de oxigênio apresenta particularidades próprias com as quais todo médico deve estar familiarizado. Uma descrição de tais detalhes está fora do escopo deste artigo, mas incentivamos fortemente o leitor a efetuar leituras adicionais de textos especializados na área.
A monitorização da oxigenação pode ser feita pela análise da $\mathrm{PaO}_{2}$ e pela $\mathrm{SaO}_{2}$. Como referido anteriormente, tais parâmetros são influenciados pela $\mathrm{FiO}_{2}$, em que o paciente está respirando, podendo-se utilizar a relação $\mathrm{PaO}_{2} / \mathrm{FiO}_{2}$ para avaliação da eficácia das trocas gasosas em diferentes ofertas de oxigênio (Tabela II).

Tabela II - Gradação da Insuficiência Respiratória Em Função da Relação PaO2/FiO2.

Superior a $400 \mathrm{mmHg}$ - Normal.

De 300 - 400 mmHg - Déficit de oxigenação.

Inferior a $300 \mathrm{mmHg}$ - Insuficiência Respiratória.

Inferior a 200 mmHg - Insuficiência Respiratória, Grave.

Em relação à oxigenoterapia, alguns aspectos devem ser salientados, como os que vêm a seguir:

- O objetivo é manter uma $\mathrm{PaO}_{2}$ acima de $60 \mathrm{mmHg}$, com a menor $\mathrm{FiO}_{2}$ possível, devido ao risco de toxicidade pulmonar por oxigênio, com o uso de $\mathrm{FiO}_{2}$ além de $60 \%$, por períodos muito prolongados.

- Um número pequeno de pacientes com DPOC, ao receber oxigênio, poderá cursar com elevações dos níveis de gás carbônico. Explicações para tal fenômeno envolvem reduções do estímulo respiratório, aumento do espaço morto por dilatação brônquica, e o deslocamento do gás carbônico ligado à hemoglobina pelo oxigênio administrado. Um número ainda menor de pacientes poderá evoluir com elevações muito acentuadas da $\mathrm{PaCO}_{2}$ e a instalação de distúrbios neurológicos, tais como sonolência e coma, caracterizando o quadro de narcose por $\mathrm{CO}_{2}$. Portanto, em tais pacientes, é necessária a repetição da gasometria arterial trinta minutos após a instalação do oxigênio e monitoração clínica, contínua, nas primeiras horas.

- O tratamento da IR ventilatória, tipo II é a instalação de ventilação mecânica. $\mathrm{O}$ uso de sistemas de administração de oxigênio poderá melhorar significantemente $\mathrm{PaO}_{2}$ devido à ausência de shunt, mas não promoverá a necessária lavagem do $\mathrm{CO}_{2}$. Há, inclusive, relatos de que, em pacientes com doenças neuromusculares, particularmente distrofias musculares, o uso do oxigênio possa agravar a retenção de gás carbônico, desencadeando quadros de narcose por gás carbônico e óbito. Como a complacên- 
cia pulmonar dos pacientes com insuficiência ventilatória, na maioria das vezes, é normal, os sistemas de ventilação não invasiva têm-se mostrado particularmente úteis.

\section{Suporte ventilatório}

Em pacientes com IR do tipo I, deve-se considerar a instalação de ventilação mecânica, quando a $\mathrm{PaO}_{2}$ mantiver-se abaixo de $60 \mathrm{mmHg}$, apesar do uso de altas $\mathrm{FiO}_{2}$. Um passo inicial, antes da entubação, nessas situações, pode ser a terapia com dispositivos do tipo CPAP (pressão positiva, contínua, nas vias aéreas). Tal equipamento consiste em máscaras faciais bem acopladas, que fornecem misturas gasosas em alto fluxo, e pressão positiva, que se mantém ao longo de toda respiração. O uso do CPAP pode levar a melhoras dramáticas da oxigenação devido a efeitos fisiológicos variados, tais como a expansão alveolar, o combate às microatelectasias e o aumento da capacidade residual, funcional. Está indicado apenas em pacientes sem comprometimento importante do nível da consciência, tendo seu uso já sido associado a complicações, tais como dilatação gasosa do estômago, com vômitos e aspiração, e mesmo a necrose de bochecha nos pontos de contato facial.

Nos últimos anos, as indicações de suporte ventilatório não invasivo através de equipamentos do tipo Bipap (Bilevel positive airway pressure) têm crescido bastante. Tais dispositivos permitem a administração de altos fluxos de gás através de máscara facial ou nasal, e a simultânea regulação das pressões inspiratórias e expiratórias de maneira independente. Desse modo, o volume corrente é gerado em função do gradiente de pressão, inspiratório e expiratório e do padrão respiratório dos indivíduos. Aparelhos desse tipo têmse mostrado bastante úteis no manuseio de pacientes com IR do tipo II. Atualmente, são indicados, inclusive, para a manutenção de pacientes que necessitam de suporte ventilatório domiciliar, crônico, tais como os portadores de moléstias neuromusculares. Dentro do contexto da IR aguda, do tipo I, tentativas de estabilização respiratória com Bipap podem ser efetuadas antes da entubação traqueal, em pacientes conscientes. Nessas situações, os melhores resultados são obtidos nos casos em que se espera rápida reversão das alterações fisiopatológicas, tais como o edema pulmonar cardiogênico, ou quando a complacência pulmonar estiver pouco alterada.

Com exceção dos casos de falência cardiorrespiratória, proteção das vias aéreas e apnéia, nos quais a necessidade de suporte ventilatório é indiscutível, as indicações de ventilação mecânica, invasiva merecem uma avaliação médica, crítica. Elas compreendem importantes alterações gasométricas, resposta inadequada ao tratamento clínico, excessivo trabalho respiratório, com evidência de fadiga da mus-

Tabela III - Indicações de Ventilação Mecânica, Classificadas em Função das Altera-ções Fisiopatológicas, Basais.

\section{\begin{tabular}{c|c|c|c|c|c|c|}
\hline Mecanismo & Valores & Ventilação
\end{tabular} \\ Normais Mecânica}

Ventilação Alveolar, Inadequada

- $\mathrm{PaCO}_{2}(\mathrm{mmHg})$

- $\mathrm{PH}$

\begin{tabular}{c|c}
$35-45$ & $>55$ \\
$7,35-7,45$ & $<7,20$
\end{tabular}

Expansão Pulmonar, Inadequada

- Volume Corrente $(\mathrm{ml} / \mathrm{kg})$

- Capacidade Vital (ml/kg)

$5-8<5$

$65-75<10$

$12-20 \quad \geq 35$

Força Muscular, Inadequada

- Pressão Inspiratória, Máxima (cm H2O)

$-80 \mathrm{a}-100 \quad \geq-20$

- Capacidade Vital (m//kg)

65-75

$<10$

- Ventilação Voluntária, Máxima (VVM; 1/min)

120-180

$<2 \mathrm{X}$ o volume minuto

Trabalho Respiratório, Aumentado

- Volume minuto (l)

- Espaço morto (VD/VT)

$5-6$

$>10$

$0,25-0,40$

$>0,6$

Oxigenação

- $\mathrm{P}(\mathrm{A}-\mathrm{a}) \mathrm{O}_{2}$ com oxigênio a $100 \%(\mathrm{mmHg})$

25-65

$>350$

$-\mathrm{PaO}_{2} / \mathrm{FiO}_{2}$ (mmHg)

$<200$ 
culatura respiratória, e a depressão do estado de consciência. Uma série de parâmetros funcionais, respiratórios podem auxiliar nessa decisão, muito embora nem sempre sejam disponíveis, em função das condições do paciente, disponibilidade de equipamento ou urgência da situação. É importante lembrar que tais parâmetros têm maior validade em casos de IR aguda (Tabela III).

Em pacientes portadores de IR crônica, tais como DPOC e ou fibrose pulmonar, terminal, que não estejam respondendo bem à terapia conservadora e ao suporte respiratório não invasivo, a decisão de entubação deve ser feita largamente em bases clínicas, incluindo aí o conhecimento acerca das condições basais do doente, o estágio evolutivo da doença, prognóstico, e os desejos expressos previamente pelo paciente e familiares.

Atualmente, existe ampla gama de respiradores com diferentes concepções de funcionamento, permitindo a administração de diferentes tipos de modalidades respiratórias. Uma análise desses aspectos foge da finalidade desta revisão e pode ser encontrada em outro texto desta revista.

Uma vez revertidas as condições precipitantes da IR, é tempo de se iniciar o desmame do aparelho. Tão importante quanto saber o momento de introduzir a ventilação mecânica é reconhecer a hora de retirá-la. Uma lista de parâmetros que orientam nesse processo está relacionada na Tabela IV. É obrigatória a avaliação diária das condi- ções que permitam iniciar o desmame. Independente dos métodos que vierem a ser usados, a rigorosa supervisão do processo pelos elementos da equipe de saúde que acompanham o paciente é o elemento mais importante para o sucesso da empreitada.

\section{Tabela IV - Parâmetros Indicativos de Sucesso da Interrupção} da Ventilação Mecânica

\section{1- Condições Clínicas}

- Presença de drive respiratório

- Nível de consciência satisfatório

- Estabilidade hemodinâmica

- Drogas vasoativas e agentes sedativos em mínimas doses

- Ausência de distúrbios hidroeletroliticos

\section{2- Parâmetros de Oxigenação}

$-\mathrm{PaO}_{2}>60 \mathrm{mmHg}$ com $\mathrm{FiO}_{2} \leq 0,4$ e PEEP $\leq 5 \mathrm{cmH}_{2} \mathrm{O}$

- Relação $\mathrm{PaO}_{2} / \mathrm{FiO}_{2}>200 \mathrm{mmHg}$

- $\mathrm{P}(\mathrm{A}-\mathrm{a}) \mathrm{O} 2$ com $\mathrm{FiO} 2=1$ inferior a $350 \mathrm{mmHg}$

- Shunt $<20 \%$

\section{3- Parâmetros de Ventilação}

- $\mathrm{PaCO}_{2} \leq 45 \mathrm{mmHg}$; valor próximo ao basal para retentores crônicos.

- Volume minuto requerido $<5-10 \mathrm{l} / \mathrm{min}$

- Capacidade de dobrar o volume minuto de repouso em uma manobra de ventilação voluntária, máxima

- Volume corrente $>5 \mathrm{ml} / \mathrm{kg}$

- Capacidade vital > 10-15 ml/kg

- Pressão inspiratória máxima $<-20$ a -30 cm $\mathrm{H}_{2} \mathrm{O}$

- Freqüência respiratória $\leq 25$ ipm

- Relação freqüência respiratória/ volume corrente $<105$ i/min/l

PÁDUA Al; ALVARES F \& MARTINEZ JAB. Respiratory failure. Medicina, Ribeirão Preto, 36: 205-213, apr./dec. 2003.

ABSTRACT - The Respiratory Failure concept deals with the presence of difficulties for the respiratory system to perform its most important function, to keep satisfactory gas exchanges. There are several causes of respiratory failure, with different clinical features. The diagnosis of respiratory failure depends on the analyses of oxygen and carbon dioxide levels in arterial blood samples. This article contains a general view about gas exchange physiology and its disorders, that are the basis for understanding the classification and mechanisms involved with the different types of Respiratory Failure. It is also emphasized general aspects dealing with therapeutic issues, such as oxygen-therapy and ventilatory support indications.

UNITERMS - Respiratory Insufficiency. Pulmonar Gas Exchange. Respiration, Artificial. 


\section{BIBLIOGRAFIA RECOMENDADA}

1 - BERNARD GR; ARTIGAS A \& BRIGHAM KL. The AmericanEuropean Consensus Conference on ARDS. Definitions, mechanisms, relevant outcomes and clinical coordination. Am J Respir Crit Care Med 149: 818-824, 1994.

2 - BONEKAT HW. Non-invasive ventilation in neuromuscular disease. Crit Care Med 14: 755-797, 1998.

3 - EVANS TW. International consensus conferences in intensive care medicine: non-invasive positive pressure ventilation in acute respiratory failure. Intensive Care Med 27: 166-178, 2001

4 - FISHMAN AP. Acute respiratory failurere. In: FISHMAN AP. Pulmonary diseases and disorders. Mc Graw-Hill, New York, V.3, p. 2185-2201, 1988.
5 - UBRAN A \& TOBIN MJ. Monitoring during mechanical ventilation. Clin Chest Med.17: 453-474, 1996.

6 - MARTINEZ JAB \& PADUAAI. Modos de assistência ventilatória. Medicina, Ribeirão Preto 34:133-142, 2002.

7 - SCALAN CL \& HEUER A. Gasoterapia medicinal. In: SCALAN CL; WILKINS RL \& STOLLER JK. Fundamentos da terapia respiratória de Egan. Manole, São Paulo, p. 761-796, 2000

8 - SOCIEDADE BRASILEIRA DE PNEUMOLOGIA E TISIOLOGIA II Consenso Brasileiro de Ventilação Mecânica. Sociedade Brasileira de Pneumologia e Tisiologia. J Pneumol. 26: 2000.

9 - TERRA FILHO J \& SANTOS E FONSECA CMC. Insuficiência respiratória. Medicina, Ribeirão Preto. 25: 416-437, 1992.

10 - WEST JB. Fisiologia respiratória moderna. $3^{\mathrm{a}}$ ed, Manole, São Paulo, 1990. 